Original Research Article

\title{
A prospective double blind randomized comparative study of safety and efficacy of Tapentadol versus Tramadol in post-operative orthopaedic patients
}

\author{
Vasavi Gedela $^{1 *}$, Sashank Gururaj ${ }^{2}$
}

\begin{abstract}
${ }^{1}$ Department of Pharmacology, ASRAM medical college, Eluru, Andhra Pradesh, India

${ }^{2}$ Senior Drug Safety Physician, Bio-clinical Safety and Regulatory Solutions, Mysore, Karnataka, India
\end{abstract}

Received: 16 September 2017 Accepted: 07 October 2017

*Correspondence to:

Dr. Vasavi Gedela,

Email: drvasavi.g@gmail.com

Copyright: (C) the author(s), publisher and licensee Medip Academy. This is an openaccess article distributed under the terms of the Creative Commons Attribution NonCommercial License, which permits unrestricted noncommercial use, distribution, and reproduction in any medium, provided the original work is properly cited.

\begin{abstract}
Background: Pain following surgery is an unwanted effect which is to be relieved for the better outcome of surgery and anaesthesia. The study was conducted to compare the efficacy and safety of Tapentadol with Tramadol for post-operative pain relief in orthopaedic surgeries.

Methods: After approval of IEC, patients of either sex aged between 18-55 years who had undergone orthopaedic surgeries at Government General Hospital, Kakinada between February 2013 to January 2015 were selected for study. It is a prospective double blind randomised active controlled parallel group comparative study. Sample size is 80 who fulfilled all criteria but there are 6 who discontinued drug in Tramadol group, so 74 were analysed. Written informed consent was taken from all patients, screening, pre anaesthetic evaluation done. Tablet Alprazolam $0.5 \mathrm{mg}$ was given at 9PM on previous day of surgery. Both Tramadol and Tapentadol were given $100 \mathrm{mg}$ orally 6 th hourly. Pain intensity was analysed by visual analogue scale. Sedation was assessed with Modified Wilson sedation scale. Safety and tolerability was assessed by side effects reported by patients. Patients were assessed at 0, 2, 4, 6, 8, 10, 12, 18, 24, 30, 36, 42 and 48 hours post-operatively.

Results: Pain reduction was higher in Tapentadol group on chi square test where $\mathrm{p}$ value is less than 0.001 .

Conclusions: Both Tramadol and Tapentadol are effective in treatment of postoperative pain. The pain reduction was higher in Tapentadol group on chi-square test where $\mathrm{p}$ value is $<0.001$. So, we can conclude from our study findings that Tapentadol is efficacious compared to Tramadol in reducing Post-operative pain. Both the drugs were well tolerated by the patients.
\end{abstract}

Keywords: Chi-square test, Modified Wilson sedation scale, Visual analogue scale

\section{INTRODUCTION}

The quest for pain relief, following surgery continues from beginning of the history of surgery. Autonomic nervous system is stimulated by pain leading to various stress responses which are deleterious to the patient. Postoperative analgesia is provided with NSAID's and Opiods in repeated doses. Ever since Friedrich Vilhelm Adam Serturner isolated Morphine from opium, many opioids like Pethidine, Pentazocine, Buprenorphine, Butorphanol, Alfentanil, Remifentanil, and Sufentanil etc each with its unique characteristics and potency were discovered. ${ }^{1}$ The list is growing on. Opioids are the most effective analgesic agents available till date for the management of moderate to severe painful conditions. Opioids relieve pain both by raising the pain threshold at the spinal cord level and more importantly, by altering the brain's perception of pain. Patients on opioids are still aware of the presence of pain, but the sensation is not unpleasant. Tramadol is a synthetic analgesic of the amino cyclohexanol group with opioidlike effects. Tramadol is now specifically recommended in musculoskeletal pain guidelines and neuropathic pain guidelines because of its efficacy, safety, and tolerability . $^{2-4}$ It has no major organ toxicity, and its use necessarily 
implies an NSAID-sparing effect. ${ }^{5-7}$ Tapentadol is a novel $\mu$-opioid receptor (MOR) agonist. Its relative efficacy compared with morphine is $88 \% .^{8}$ Tapentadol acts by monoamine reuptake inhibition along with MOR agonism. These actions may additively or even synergistically enhance the analgesic efficacy and/or attenuate the side effects of MOR agonists by reducing the requirement for MOR activation. The present study was conducted to: 1 . Compare the Efficacy of Tapentadol versus Tramadol given orally for post-operative pain relief in Orthopaedic surgeries. 2. Compare the safety of Tapentadol versus Tramadol and occurrence of side effects of both drugs.

\section{METHODS}

Patients of either sex aged between 18-55 years who have undergone Orthopaedic surgeries at Government General Hospital, Kakinada were selected for this study. Prior permission was taken from the Institutional Ethics Committee to conduct the study. Study Design: It is a Prospective Double blind Randomised active controlled parallel group comparative study. Study period: From February 2013 to January 2014 i.e. 12 months Sample size: After screening of 186 patients, 80 patients who fulfil the inclusion criteria were recruited after giving the Informed consent form. Sample size of 80 patients was taken depending on the previous records of orthopaedic surgeries which were around 120 surgeries per year. In this study 80 patients fulfilling all the criteria were enrolled but there were 6 patients who didn't continue treatment in Tramadol group, so after excluding those 74 patients were analysed. The drugs were chosen randomly and were allocated to the patients, so that at the end of the study there will be two groups with 40 patients in each.

\section{Inclusion criteria}

- $\quad$ Patients aged >18years and < 55years, of either sex were included

- American Society of Anaesthesiologist classification of pain grade 1 or 2

- Orthopaedic surgeries.

\section{Exclusion criteria}

- Known hypersensitivity to any one of the test drugs of the study

- with coagulopathies or on anti-coagulant drugs

- Known hypertension, diabetes mellitus, asthma, cardiac diseases

- Impaired renal (or) hepatic functions

- Taking opioid drugs (or) other analgesic drugs for any ailment

- Systemic infections or raised intra cranial pressure or any spinal defects, or having local (lumbar) dermatological lesions

- Pregnant and lactating women.

Study drugs and dosage: the following study drugs (equipotent doses) were given orally to patients in Tablet form which are labelled as A and B and given to patients according to Randomisation. Tapentadol- $100 \mathrm{mg} 6^{\text {th }}$ hourly, Tramadol- $100 \mathrm{mg} 6^{\text {th }}$ hourly. Informed written consent was obtained from all the patients after a detailed explanation and reassurance of the procedure to be performed. The screening investigations done were: Blood: hemogram, blood glucose, blood urea, serum creatinine, serum glutamic oxaloacetic transaminase, serum glutamic pyruvic transaminase. Urine: analysis of urine for- albumin, sugar, microscopy, chest X-ray, electrocardiogram, ultrasound scan- abdomen. pre anaesthetic evaluation was done. All patients were premedicated with Tab. Alprazolam $0.5 \mathrm{mg}$ at $9.00 \mathrm{pm}$ on previous day of surgery. The drugs, Tapentadol 100mg and Tramadol $100 \mathrm{mg}$ medications were labelled as A and B, this was double blinded so that both drug administrator i.e. staff nurse and patient don't know the content of it. Then they were selected randomly using Random table method for that particular patient.

In the recovery room/post-operative ward linear Visual Analogue Scale (VAS) consisting of $100 \mathrm{~mm}$ line with $0=$ No pain and $100=$ Worst possible pain was explained to all the patients. During analysis the Visual Analogue Scale was categorised into 0- $20 \mathrm{~mm}$ as No pain, 21-40 as Mild pain, 41-60 as Moderate pain, 61-100mm severe pain. The test drug was administered orally and the Group to which the patients were assigned was recorded. Time of administration of study drug was recorded as zero hour. Patients were asked if there was even a slight decrease in pain on Visual analogue scale following the administration of the drug and corresponding time was recorded. The score on VAS was evaluated at $0,2,4,6,8,10,12,18,24$, $30,36,42$ and 48 hours post operatively.

Sedation was assessed with Modified Wilson Sedation Scale which consists of 5 scores ranging from 1-5, 1 is fully awake and oriented, 2-drowsy, 3- Eyes closed but arousable to command, 4- Eyes closed but arousable to mild physical stimulation (earlobe tug), 5 - eyes closed and unarousable to mild physical stimulation. The pulse rate, blood pressure and were recorded every 2 hours and subjects were monitored for side effects like nausea, vomiting was noted down. Primary outcome measures: 1) Pain intensity score (measured on VAS) was measured was measured at $0,2,4,6,8,10,12,18,24,30,36,42$ and $48 \mathrm{hrs}$ post operatively. 2) Pain relief was assessed on 6hours, 24hours and 48hours after surgery. Secondary outcome measures: 1. Modified Wilson Sedation Scale was used to assess the dizziness during the course of postoperative period. 2. Safety was assessed based on the number of patients experiencing side effects during and after the treatment. Statistical analysis was done for 40 patients in Tapentadol group and 34 patients in Tramadol group for both primary and secondary outcome variables. The following assumptions on data were made: 1. Dependent variables should be normally distributed. 2 . Samples drawn from the population should be random. 3 . Cases of the samples should be independent. Descriptive statistical analysis was carried out in the present study. 
Results on continuous measurements are presented as Mean \pm SD and results on categorical measurements are presented as number (\%). Chi square test was employed to test for difference in pain at 24 hours and 48hours, $\mathrm{P}$ - value with $5 \%$ level of significance and odds ratio with $95 \%$ Confidence interval were calculated. Proportion of patients reporting side effects in both arms was tested employing Chi square test. Statistical software: statistical software namely SPSS 22.0 was used for analysis of the data.

\section{RESULTS}

A total of 80 participants were enrolled in our study and given the test drugs during the study time. This population has been selected from the total of 186 patients who had under gone the different surgeries in orthopaedics department. Of the 80 participants 40 were given Tramadol $100 \mathrm{mg}$ orally and for other 40 patients it was Tapentadol $100 \mathrm{mg}$ orally. As shown in Table 1, at 6 hours after surgery which was considered as baseline, 17 patients $(42.5 \%)$ had shown moderate pain and 23 patients $(57.5 \%)$ had suffered with severe postoperative pain in Tapentadol intervention group. 14 patients $(35 \%)$ with moderate pain and 26 patients $(65 \%)$ with severe pain in Tramadol intervention group. At 24 hours of surgery 40 patients $(100 \%)$ in Tapentadol intervention group had shown mild pain, and it was 22 patients $(56.4 \%)$ in Tramadol intervention group, remaining 17 patients (43.6\%) in Tramadol intervention group had shown moderate pain.

Table 1: Distribution of pain on visual analogue scale (VAS).

\begin{tabular}{|c|c|c|c|c|}
\hline Time interval & $\begin{array}{l}\text { No. of patients in } \\
\text { Tapentadol group }\end{array}$ & $\begin{array}{l}\text { No. of patients in Tramadol } \\
\text { group }\end{array}$ & P-value & Odds ratio \\
\hline \multicolumn{5}{|l|}{6 hours } \\
\hline Moderate pain & $17(42.5 \%)$ & $14(35 \%)$ & 0.25 & $1.37(0.55-3.38)$ \\
\hline Severe pain & $23(57.5 \%)$ & $26(65 \%)$ & & \\
\hline \multicolumn{5}{|l|}{24 hours } \\
\hline Mild pain & $40(100 \%)$ & $22(56.4 \%)$ & $<0.001$ & Can't be calculated \\
\hline Moderate pain & $0(0 \%)$ & $17(43.6 \%)$ & & \\
\hline \multicolumn{5}{|l|}{48 hours } \\
\hline No pain & $30(75 \%)$ & $7(20 \%)$ & $<0.001$ & $12.0(4.01-35.9)$ \\
\hline Mild pain & $10(25 \%)$ & $28(80 \%)$ & & \\
\hline
\end{tabular}

Missing data: 1 subject didn't take the medicine at 24hours, 5 subjects didn't take the drug at 48hours in Tramadol group.

Table 2: Comparison of adverse effects between the two study groups.

\begin{tabular}{|lllll|}
\hline Adverse effect & $\begin{array}{l}\text { No. of patients in } \\
\text { Tapentadol group }\end{array}$ & $\begin{array}{l}\text { No. of patients in } \\
\text { Tramadol group }\end{array}$ & P value & $\begin{array}{l}\text { Odds ratio } \\
\text { (95\% C.I) }\end{array}$ \\
\hline Nausea & & & & \\
\hline No & $33(82.5 \%)$ & $25(62.5 \%)$ & 0.045 & $2.83(1.01-7.98)$ \\
\hline Yes & $7(17.5 \%)$ & $15(37.5 \%)$ & & \\
\hline Vomiting & $35(87.5 \%)$ & $31(77.5 \%)$ & 0.24 & $2.032(0.62-6.72)$ \\
\hline No & $5(12.5 \%)$ & $9(22.5 \%)$ & & \\
\hline Yes & & & & \\
\hline Dizziness* & $31(77.5 \%)$ & $23(57.5 \% 0$ & 0.15 & \\
\hline Fully awake and oriented & $6(15 \%$ & $10(25 \%)$ & & \\
\hline Drowsy & $3(7.5 \%)$ & $7(17.5 \%)$ & & \\
\hline Eyes closes but arousable to command & & & \\
\hline
\end{tabular}

*Dizziness according to modified Wilson sedation scale.

The pain reduction was higher in Tapentadol group on chisquare test where $\mathrm{p}$ value is $<0.001$, here the odds ratio couldn't have calculated (because there were no patients with moderate pain in Tapentadol group). At 48 hours of surgery 30 patients $(75 \%)$ in Tapentadol intervention group had shown no pain, and it was 7 patients $(20 \%)$ in Tramadol intervention group. 10 patients $(25 \%)$ in Tapentadol intervention group had shown mild pain, and it was 28 patients $(80 \%)$ in Tramadol intervention group. The pain reduction was higher in Tapentadol group on chisquare test where $\mathrm{P}$ - value is $<0.001$.

Here the odds ratio was 12 with $95 \%$ confidence interval of 4.01-35.9. Table 2 and Figure 1, 2, 3 compares the adverse effects between two groups. Seven patients $(17.5 \%)$ had shown Nausea with Tapentadol intervention group, and it was 15 patients $(37.5 \%)$ in Tramadol intervention group. 


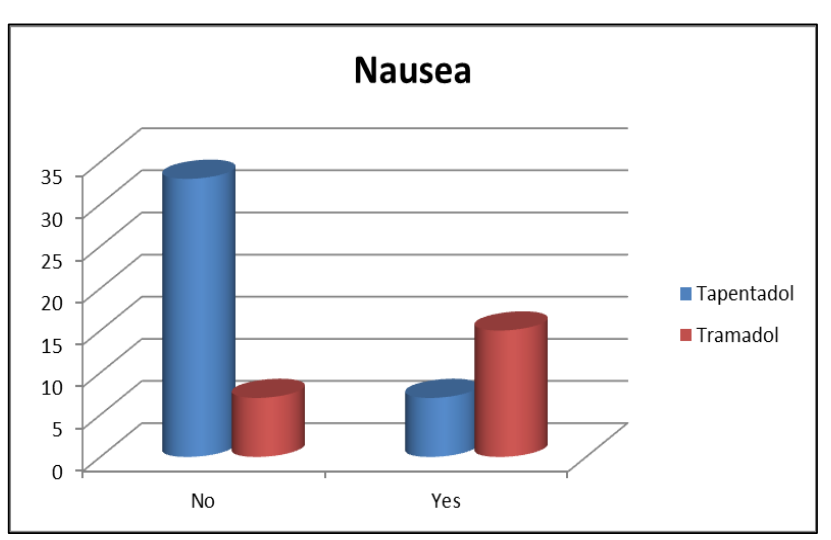

Figure 1: Comparison of adverse effects nausea between the two study drugs.

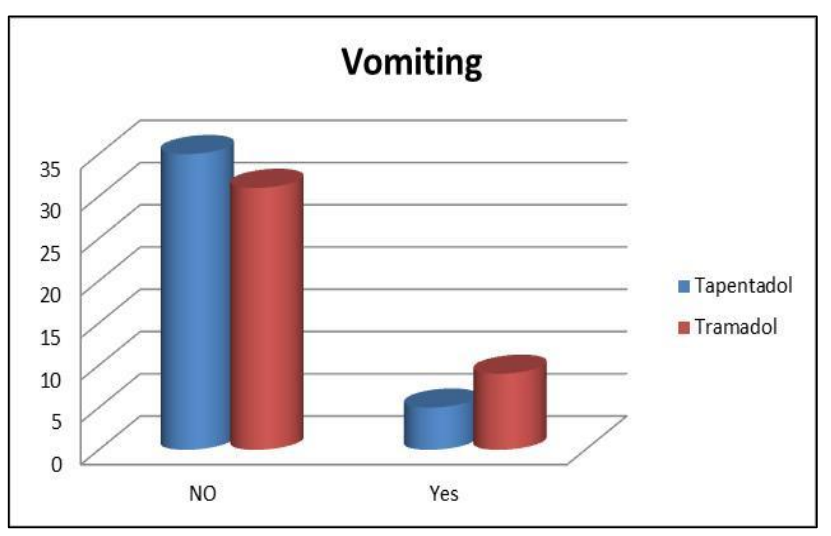

Figure 2: Comparison of adverse effect vomiting between two groups.

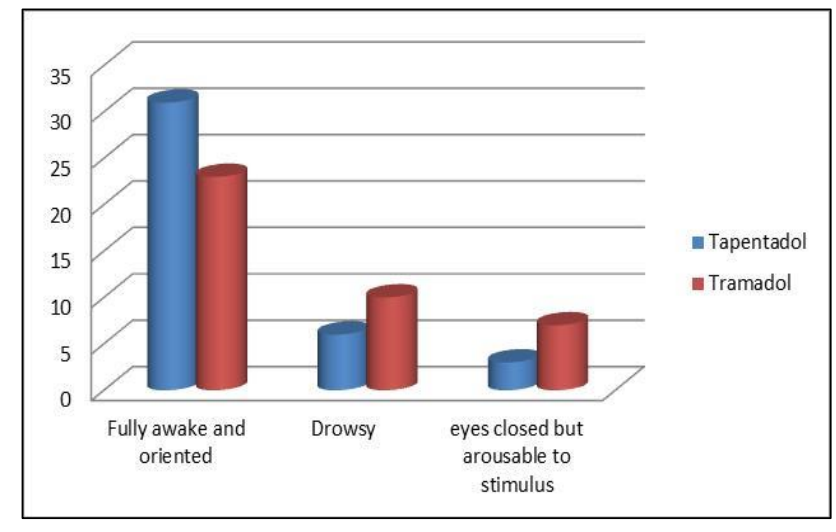

Figure 3: Adverse effect dizziness between two groups.

On chi square test it was found that the tramadol drug is associated with more nausea compared to Tapentadol, where the $\mathrm{P}$ - value is $0.045(\mathrm{OR}=2.83,95 \%$ C.I. 1.01 7.98). It was found that other adverse effects i.e. vomiting and dizziness were similar in both groups.

And there were no significance association found on chi square test. It was about 5 patients $(12.5 \%)$ had shown vomiting with Tapentadol intervention group, and it was 9 patients $(22.5 \%)$ in Tramadol intervention group. The Pvalue is 0.24 . The odds ratio was 2.032 (95\% C.I. 0.626.72). Nine patients $(22.5 \%)$ had shown Dizziness with Tapentadol intervention group, and it was with 17 patients $(42.5 \%)$ in Tramadol intervention group. The P-value is 0.06 (OR-2.55, 95\% C.I. 0.96-6.73).

Table 3: Age distribution of study groups.

\begin{tabular}{|ll|ll|}
\hline Age (years) & No. of patients in Tapentadol group & No. of patients in Tramadol group & Total \\
\hline $18-25$ & 14 & 5 & 19 \\
\hline $26-35$ & 10 & 9 & 19 \\
\hline $36-45$ & 12 & 11 & 23 \\
\hline $46-55$ & 4 & 15 & 19 \\
\hline Total & 40 & 40 & 80 \\
\hline Mean & 32.62 (SD-9.7) & 39.27 (SD-10.2) & 35.95 (SD-10.47) \\
\hline
\end{tabular}

Table 4: Gender distribution of study subjects.

\begin{tabular}{|ll|ll|}
\hline Gender & Tapentadol & Tramadol & Total \\
\hline Female & $13(32.5 \%)$ & $19(47.5 \%)$ & $32(40.0 \%)$ \\
\hline Male & $27(67.5 \%)$ & $21(52.5 \%)$ & $48(60.0 \%)$ \\
\hline Total percentage & $40(100 \%)$ & $40(100 \%)$ & $80(100.0 \%)$ \\
\hline
\end{tabular}

\section{DISCUSSION}

In this study, a potential treatment effect was evaluated on changes in pain intensity determined by spontaneous patient self-report using a standard validated unimodal rating scale (VAS). Paper by Collins. SL et al reported that a mean of $49 \mathrm{~mm}$ on corresponding VAS scores had shown that the patient was suffering from pain. ${ }^{9}$ Based on the above study and other studies we have categorised our VAS scale into no pain, mild, moderate and severe pain as explained in methodology. In this study, the mean Tapentadol and Tramadol dosage was 400 mg/24 hours. 
Both treatments were well tolerated with only six patients in the Tramadol group (15\%) withdrawing from the study because of intolerance to the drug and their personal reasons so that they don't want to take the pills we have provided. The study demonstrated that Tapentadol had more effectiveness in reducing post-operative pain on Visual Analogue Scale compared to Tramadol. This is in contrast to previous studies where they have compared tramadaol and other opiods to NSAIDs like diclofenac in reducing the pain. For instance in a study conducted by Courtney MJ, et al, in 2001 comparing Tramadol versus Diclofenac for post-tonsillectomy analgesia, they found that average visual analogue scale for pain scores for 14 days did not differ significantly (Diclofenac group:mean, 38.4 (17.5) Versus Tramadol group: mean, 37.8 (15.6). ${ }^{10}$ In a study conducted by Wade WE et al, Tapentadol is well tolerated and effective analgesic for the treatment of moderate to severe acute pain. Tapentadol has been reported to be effective in osteoarthritis and low back pain. ${ }^{11}$

The most common adverse events in both groups (nausea and dizziness) were considered to be treatment-related, probably since investigator was aware of the safety profile of both drugs. On chi square test it was found that the Tramadol drug is associated with more nausea compared to Tapentadol. It was found that other adverse effects i.e. vomiting and dizziness were similar in both groups. And there were no significance association. It was observed that the study subjects did not experience any prolonged side effects during their recovery period. In a study conducted by Etropolski $\mathrm{M}$ et al, to compare the analgesic efficacy and adverse effects between Oxycodone and Tapentadol proved that Tapentadol produced significantly lower incidence of gastrointestinal adverse events such as nausea, vomiting, and constipation compared to Oxycodeine and Tapentadol was not inferior to Oxycodone in analgesic efficacy. But there were no studies where Tapentadol and Tramadol compared for the adverse effects and had shown that Tapentadol had better tolerability than Tramadol. ${ }^{12}$

\section{Limitations}

We excluded patients who are diabetics, Hypertension and other chronic diseases in order to limit inclusion of refractory patients who could make the trial less sensitive. It is also true that one single patient may present several different features of post-operative pain simultaneously because of difference in the type of trauma or surgery. In this study, there has been no attempt to evaluate the different pain symptoms individually. In Tramadol group 6 patients didn't continue the treatment till 48 hours. This might be one of the reasons for strong association of Tapentadol with reduction in pain on VAS score. And also in Tramadol group the age is much higher than Tapentadol group which might be one more reason for the much stronger association i.e. effectiveness of Tapentadol is higher than Tramadol. As the age increases the metabolism of the drug get affected.

\section{External validity}

As the study patients in two groups were different in age pattern and the study setting and design is different the results cannot be compared to other study settings so external validity is limited.

\section{CONCLUSION}

From the results of the present study it can be concluded that both Tapentadol and Tramadol are effective in the treatment of Postoperative pain. The pain reduction was higher in Tapentadol group on chi-square test where $p$ value is $<0.001$. So, we can conclude from our study findings that Tapentadol is efficacious compared to Tramadol in reducing Post-operative. Both the study drugs were well tolerated.

\section{ACKNOWLEDGEMENTS}

Authors would like to thank teachers and the patients.

Funding: No funding sources

Conflict of interest: None declared

Ethical approval: The study was approved by the Institutional Ethics Committee

\section{REFERENCES}

1. Nicolaou KC, Montagnon T. Molecules that changed the world. Molecules that changed the World. 2008. Available at https://www.ncbi.nlm.nih.gov/pmc/articles/PMC3500 $237 /$.

2. Hollingshead J, Dühmke R, Cornblath D. Tramadol for neuropathic pain. Cochrane Database Systematic Reviews. 2005;3:CD003726.

3. Dworkin RH, Backonja M, Rowbotham MC, Allen RR, Argoff CR, Bennett GJ, et al. Advances in neuropathic pain: diagnosis, mechanisms, and treatment recommendations. Arch Neurol. 2003;60(11):1524-34.

4. Finnerup N, Otto M, McQuay H, Jensen T, Sindrup $\mathrm{SH}$. Algorithm for neuropathic pain treatment: an evidence based proposal. Pain. 2005;118(3):289-305.

5. Raffa R. Pharmacological aspects of successful longterm analgesia. Clin Rheumatol. 2006;25(1):9-15.

6. Shipton E. Tramadol-present and future. Anaesthesia Intensive Care. 2000;28(4):36374.

7. Schnitzer TJ, Kamin M, Olson WH. Tramadol allows reduction of naproxen dose among patients with naproxen responsive osteoarthritis pain: a randomized, double blind, placebo controlled study. Arthritis Rheumatism. 1999;42(7):1370-7.

8. Tzschentke TM, Christoph T, Kögel B, Schiene K, Hennies HH, Englberger W, et al. (-) -(1R, 2R)-3-(3Dimethylamino-1- ethyl-2-methyl-propyl)-phenol Hydrochloride (Tapentadol $\mathrm{HCl}$ ): a Novel $\mu$-Opioid Receptor Agonist/Norepinephrine Reuptake Inhibitor with Broad-Spectrum Analgesic Properties. J 
Pharmacol Experimental Therapeut. 2007;323(1):26576.

9. Collins SL, Moore RA, McQuay HJ. The visual analogue pain intensity scale: what is moderate pain in millimetres? Pain. 1997;72(1):95-7.

10. Courtney MJ, Cabraal D. Tramadol vs diclofenac for posttonsillectomy analgesia. Arch Otolaryngol Head Neck Surg. 2001;127(4):385-8.

11. Wade WE, Spruill WJ. Tapentadol hydrochloride: a centrally acting oral analgesic. Clin Therapeut. 2009;31(12):2804-18.
12. Etropolski M, Kelly K, Okamoto A, Rauschkolb C. Comparable efficacy and superior gastrointestinal tolerability (nausea, vomiting, constipation) of tapentadol compared with oxycodone hydrochloride. Advances Therapy. 2011;28(5):401-17.

Cite this article as: Gedela V, Gururaj S. A prospective double blind randomized comparative study of safety and efficacy of Tapentadol versus Tramadol in post-operative orthopaedic patients. Int $\mathbf{J}$ Basic Clin Pharmacol 2017;6:2727-32. 